\title{
Isolation of one Compound from of Syzygium cumini L. Leaves Responsible for UV Radiation Absorption
}

\author{
Prasenjit Mitra ${ }^{1}$, Prasanta Kumar Mitra ${ }^{2 *}$, Tanaya Ghosh ${ }^{2}$ \\ ${ }^{1}$ Department of Biochemistry, All India Institute of Medical Sciences (AIIMS), Jodhpur, India \\ ${ }^{2}$ Department of Medical Biotechnology, Sikkim Manipal University, Sikkim, India \\ *Corresponding Author: Prasanta Kumar Mitra, Professor and Head, Department of Medical Biotechnology, Sikkim Manipal University, \\ Sikkim, India.
}

Received: April 17, 2019; Published: May 30, 2019

DOI: $10.31080 /$ ASPS.2019.03.0292

\begin{abstract}
Since long Syzygium cumini Linn. (S. cumini L.) is being used for treatment of several diseases. In Ayurveda the plant is used to pacify kapha and pitta dosha. It is also used as tonic for weakness, to treat problems of gum, teeth, eye, skin as well as to correct anemia and sexual weakness. Modern researches explored a wide range of pharmacological properties of this plant. These include anti diabetic, anti microbial, anti fertility, anti cancer, anti inflammatory, anti oxidant, anti gastric ulcer, hepato protective, gastro protective etc. Recently we have noted UV absorption property of $S$. cumini L. leaves and maximum absorption was found during rainy season. In the present work we tried to isolate the active compound from the plant leaves responsible for UV radiation absorption. $S$. cumini L. leaves were processed for isolation work by standard methods. Solvent extraction and acid hydrolysis were done followed by solvent treatment, chromatographic experiments. Finally a compound was crystallized. UV absorption property of the isolated compound was studied. The compound showed maximum absorption at $200 \mathrm{~nm}$. The compound, therefore, may be used to protect humans from UV radiation absorption.
\end{abstract}

Keywords: Syzygium cumini Linn. Leaves; UV Absorbing Property; Isolation of Active Compound; Sunscreen Lotion

\section{Introduction}

S. cumini L. (family, Myrtaceae) is a large evergreen tree and a tropical fruit tree of great economic importance. Commonly known as Jambul tree the plant is found almost everywhere in India, Bangladesh, Nepal, Pakistan and Indonesia [1].

S. cumini L. contains several phytochemicals. Myricetin, ellagitannin, nilocitin, 3-0-ß-D-glucaronopyranoside n-hepatcosane, kaempferol, and aminoacids like alanine, glycine etc. are present in leaves. Stem bark contains, n-octacosanol, n-triacontanol, $ß$-sitosterol-D-glucoside, astragalin, betulinic acid, crategolic (maslinic) acid, soxalic acid, citric acid, betulinic acid, $ß$-sitosterol, n-nonacosane, quercetin, myricetin, sitosterol, glycolic acids, n-hentriacontane, kaempferol-3-o glucoside, eugenin, friedelin, epi-friedelanol and gallic acid. Flower has quercetin, kaempferol, erategolic acid (maslinic acid), Oleanolic acid, and myricetin flavonoids -isoquercitrin $[2,3]$.

S. cumini L. is a potential source of nutraceuticals [4]. The plant has wide range of pharmacological properties. Bark and pulp of the plant are efficacious for diabetes; fruit is anti hyper lipidemic, possessing anti cancer property; seeds exert anti inflammatory and anti gastric ulcer activity; leaf has anti viral, anti allergic, anti bacterial, anti diabetic, anti oxidant and anti DNA damage activities [5].

Recently we have seen that $S$. cumini L. leaf has UV absorption activity and maximum activity was found during rainy season $[6,7]$. Therefore, aim of the present work was to isolate the active compound present in leaves of S. cumini L. responsible for UV absorption property..

\section{Materials and Methods \\ Plant material}

S. cumini L. leaves were purchased from the local market during rainy season. Leaves were authenticated by the taxonomist of the department of Botany of the University of North Bengal, Siliguri. A voucher specimen was kept in the department of Medical Biotechnology, Sikkim Manipal Institute of Medical Sciences of Sikkim Manipal University, Gangtok, Sikkim, India for future references. 


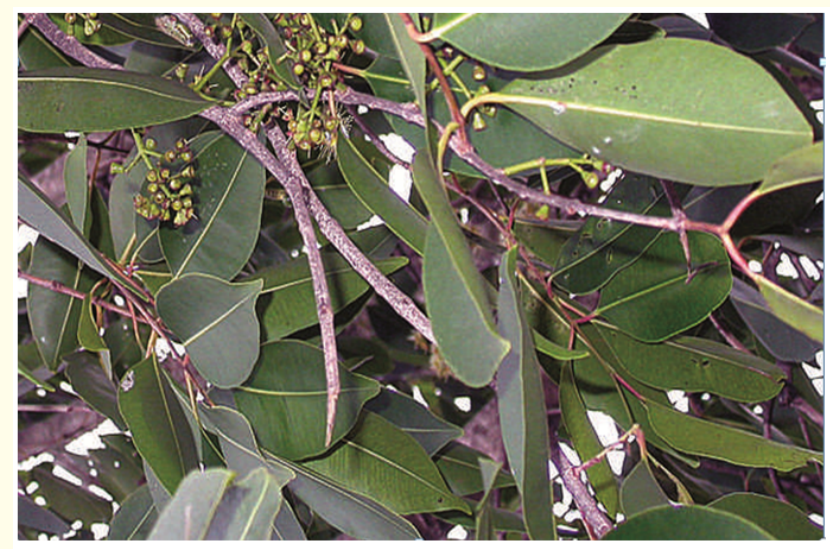

Figure 1: S. cumini L. leaves.
Preparation of the plant leaves

S. cumini L. leaves were washed thoroughly under running tap to remove dust and then by distilled water. Leaves were shade dried and powdered. The powder was used for isolation study.

\section{Chemicals}

Chemicals required for the study were purchased from Himedia Lab and Loba Chem. Lab, India as well as from Merck, Germany.

Isolation of active compound

This was carried out by the following steps. Principles of standard isolation procedures of chemical compounds from plant sources are applied [8-11].

Powdered leaves of S. cumini L. (50 g)

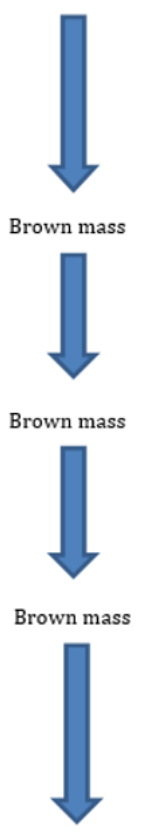

SOLVENT EXTRACTION

Extracted with $500 \mathrm{ml}$ of acetone in sexhlet at $37^{\circ} \mathrm{C}$ for 20 minutes.

It was then centrifuged. Supernatant was evaporated to dryness.

\section{Third band was found active}

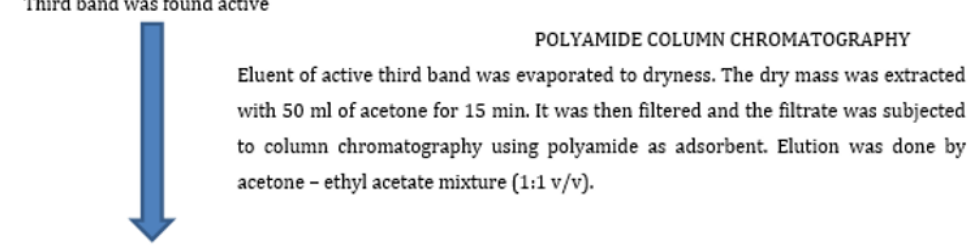

ACID REFLUX

Refluxed with $50 \mathrm{ml}$ of $1(\mathrm{~N}) \mathrm{HCl}$ for $15 \mathrm{~min}$ on a water bath at $100^{\circ} \mathrm{C}$. It was then cooled and centrifuged. Supernatant was evaporated to dryness.

TREATMENT WITH ETHYL ACETATE

Extracted with $50 \mathrm{ml}$ of ethyl acetate on a rotary shaker for $15 \mathrm{~min}$. It was then centrifuged. Supernatant was evaporated to dryness.

ALUMINA COLUMN CHROMATOGRAPHY

Brown mass was extracted with $50 \mathrm{ml}$ of acetone for $15 \mathrm{~min}$. It was then filtered. With filtrate alumina column chromatography was performed. Elution was done by acetone - ethyl acetate mixture $(1: 1 \mathrm{v} / \mathrm{v})$.

Fourth band was found active

\section{SILICA GEL G CHROMATOGRAPHY}

Eluent of active fourth band was evaporated to dryness. The dry mass was extracted with $50 \mathrm{ml}$ of acetone for $15 \mathrm{~min}$. It was then filtered. With filtratel silica gel $\mathrm{G}$ column chromatography was done. Elution was by acetone - ethyl acetate mixture $(1: 1 \mathrm{v} / \mathrm{v})$.

First band was found active

\section{CRYSTALLIZATION}

Eluent of the active first band obtained from the above step was evaporated to dryness. Repeated crystallization was done from $\mathrm{n}$ - benzene, chloroform mixture $(40: 60, v / v)$.

Crystals obtained (7.1 mg)

Figure a 
UV absorption property of the isolated compound

Distilled water $(100 \mathrm{ml})$ was added to $10 \mathrm{mg}$ of the isolated compound. The solution was filtered and the filtrate was processed in a spectrophotometer for UV ray absorption at the ranges of 200$400 \mathrm{~nm}$ at $10 \mathrm{~nm}$ intervals.

\section{Results and Discussion}

\section{Isolation of the compound}

Brown coloured compound was isolated.

\section{UV absorption property of the isolated compound}

Isolated compound absorbed UV ray in all wave lengths of UV region. Absorptions at $400 \mathrm{~nm}, 350 \mathrm{~nm}, 300 \mathrm{~nm}, 250 \mathrm{~nm}$ and 200 nm were, $0.37,0.51,0.69,0.95$ respectively.. Maximum absorption, however, was noted at $200 \mathrm{~nm}$ (1.6). Results are summarized in figure 2 .

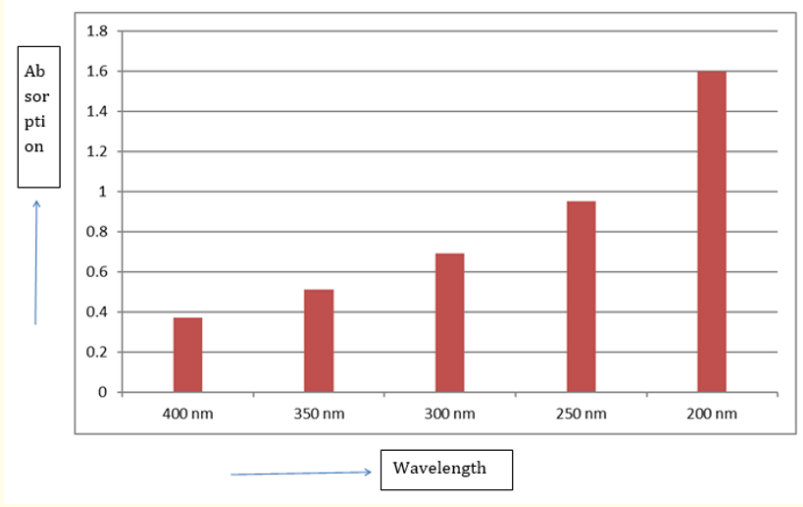

Figure 2: UV radiation absorption by the isolated compound from S. cumini L. leaves.

Ultraviolet radiation is non-ionizing radiation. It falls under 180 - $400 \mathrm{~nm}$ wavelength region of electromagnetic spectrum. Depending on wavelength ultraviolet radiation is divided into three categories: UV - A, UV - B and UV - C. UV-A having the longest wavelength 315-400 nm, known as black light, has least energetic photons. On the other hand, UV - C having the shortest wavelength 100 $280 \mathrm{~nm}$, known as germicidal has highest energetic photons. UV - B, having wavelength 281-314 nm, known as erythemal, however, falls in the intermediate category. Ultraviolet radiation also generates from laboratory instruments like germicidal lamps, biological safety cabinets, cross linkers, lasers, trans illuminators etc. However, the major source of UV ray is solar radiation or sunlight [12].
Solar UV-radiation has good effect on humans. It is required for cutaneous synthesis of vitamin D. This synthesis covers almost $90 \%$ of the vitamin D requirement of human body. UV radiation has bad effect too and the effects are plenty. These include skin and eye injury, genetically determined photo sensitivities, photosensitivity reactions to ingested drugs, etc. It has been observed that if a person is under excessive exposure to UV rays then it causes atrophy, wrinkling, pigmentary changes and malignancy. Three types of skin cancer may develop. These are squamous cell carcinoma, basal cell carcinoma and malignant melanoma. Eye is also affected. UV radiation can cause injury to cornea and there is painful inflammation of eye. If eye gets chronic UV exposure, it can lead to the formation of cataracts. It was also observed that over-exposure to UV radiation can change the distribution and function of WBC in humans causing harmful suppressing effect on the immune system [13].

Therefore, identification of sources to absorb UV radiation from the environment is needed for human protection. Efforts are going on. Research in this direction has been extended even in the field of medicinal plants. Several medicinal plants like Phyllostachys pubescens, Calotropis gigantea, Mentha piperita, Azadirachta indica, Carica papaya, Lycopersicon esculantum, Aloe vera, Oscimum sanctum, etc. are now known to have anti solar activity $[14,15]$.

In the present study we have isolated a compound from $S$. cumini L. leaves. The compound can absorb UV radiation in all wavelength region but maximum absorption was at found $200 \mathrm{~nm}$. The compound now needs characterization. Work in this direction is presently going on in our laboratory.

\section{Conclusion}

In the present study we found UV radiation absorption property of the isolated compound from $S$. cumini L. leaves. The property may be utilized in future to protect humans from UV radiation.

\section{Recommendation}

Isolated compound from $S$. cumini L. leaves may be used in preparation of sunscreen lotion as UV absorbing material.

\section{Acknowledgements}

We gratefully acknowledge the cooperation of taxonomists of the department of Botany, University of North Bengal, Siliguri, Dist. Darjeeling, West Bengal for identification of S. cumini L. leaves.

\section{Conflict of interest}

Nil 


\section{Bibliography}

1. Jadhav VM., et al. "Herbal medicine: Syzygium cumini: A Review". Journal of Pharmacy Research 2.8 (2009): 1212-1219.

2. Bhargava KK., et al. "Chemical components of Eugenia jambolana stem bark". Current Science 43.20 (1974): 645- 646.

3. Gupta GS and Sharma DP. "Triterpenoid and other constituents of Eugenia jambolana Leaves". Phytochemistry 13 (1974): 2013-2014.

4. Chaudhary B and Mukhopadhyay K. "Syzygium cumini (L.) skeels: A potential source of nutraceuticals". International Journal of Pharmacy and Biological Sciences 2.1 (2012): 46-53.

5. Pradhan Madhulika. "Phytochemistry, Pharmacology and Novel Delivery Applications of Syzygium cumini (L.)". IJPPR 7.1 (2016): 659-675.

6. Mitra Prasenjit., et al. "UV absorption property of Syzygium cumini l. leaves". Acta Scientific Pharmaceutical Sciences 2.9 (2018): 69-73.

7. Mitra Prasenjit., et al. "Effect of season on UV absorption property of Syzygium cumini l. leaves". Acta Scientific Agriculture 2.11 (2018): 59-63.

8. Cannell RJP. "Natural Products Isolation. New Jersey". Human Press Inc. (1998): 165-208.

9. Li HB., et al. "Separation methods used for Scutellaria baicalensis active components". Journal of Chromatography 8.12 (2004): 277-290.

10. Huie CW. "A review of modern sample-preparation techniques for the extraction and analysis of medicinal plants". Analytical and Bioanalytical Chemistry 373 (2002): 23-30.

11. Sasidharan S., et al. "Extraction, Isolation and Characterization of Bioactive Compounds from Plants' Extracts". African Journal of Traditional, Complementary and Alternative Medicines 8.1 (2011): 1-10.

12. D'Orazio., et al., "UV Radiation and the Skin". International Journal of Molecular Sciences 14 (2013): 12222-12248.

13. MacKie RM. "Effects of Ultraviolet Radiation on Human Health". Radiation Protection Dosimetry 91.1-3 (2000): 15-18.
14. Gupta Dipali. "Absorbing Properties of Some Plant Derived Extracts". Research Journal of Chemical and Environmental Sciences 1.2 (2013): 34-36.

15. Gupta Vandana., et al. "Environment friendly antibacterial and uv protective finish on cotton using S. cumini (l.) leaves extract". International Journal of Textile and Fashion Technology 7.1 (2017): 53-62.

Volume 3 Issue 6 June 2019

(C) All rights are reserved by Prasanta Kumar Mitra., et al. 